\title{
On the Convergence of a Nonlinear Boundary-Value Problem in a Perforated Domain
}

\author{
Yulia Koroleva \\ Department of Higher Mathematics, Gubkin Russian State University of Oil and Gas, Leninsky Prospect 65-1, \\ Moscow 119991, Russia \\ Correspondence should be addressed to Yulia Koroleva; korolevajula@mail.ru
}

Received 16 July 2015; Accepted 7 September 2015

Academic Editor: Elena I. Kaikina

Copyright (C) 2015 Yulia Koroleva. This is an open access article distributed under the Creative Commons Attribution License, which permits unrestricted use, distribution, and reproduction in any medium, provided the original work is properly cited.

\begin{abstract}
We consider a family with respect to a small parameter of nonlinear boundary-value problems as well as the corresponding spectral problems in a domain perforated periodically along a part of the boundary. We prove the convergence of solution of the original problems to the solution of the respective homogenized problem in this domain.
\end{abstract}

\section{Introduction}

The paper is devoted to study of convergence of nonlinear boundary-value problems in a domain perforated along the boundary. There exist a lot of literatures, where boundaryvalue problems in perforated domains were studied. We refer to works [1-33]. In these papers and monographs the authors studied different kinds of perforation for linear as well as for nonlinear differential operators. Usually it is considered a family of problems depending on small parameter that characterizes the size of perforation. The main goal of the research is to find a homogenized (limit) model which is close to originally considered problems posed in the perforated domain. The general technique of homogenization method can be found in $[23,24,27,28]$.

The present paper will deal with convergence of boundary-value problems in perforated domains for nonlinear $p$ Laplace operator. Some problems for nonlinear operators were homogenized, for example, in [1, 20, 21, 30-33]. We consider a family of boundary-value problems in $n$-dimensional domain, $n>2$, which is periodically perforated along the boundary by small sets. It is assumed that the diameter of each set and the distance between them have the same order. In our problem we suppose that the Dirichlet condition holds on the boundary of cavities, while the Naumann boundary condition is fulfilled on the boundary of the domain. We derive the limit (homogenized) problem for the original problems when the small parameter characterizing the size of perforation tends to zero. Moreover, we establish the strong convergence in $W^{1, p}$ of the solutions for the considered problems to the corresponding solution of the limit problem. In addition we have obtained an estimate of the solution in a neighborhood of the eigenvalue of a corresponding spectral problem.

One of our goals is to prove the asymptotic behavior for the eigenvalue problem for $p$-Laplace operator in our perforated domain. Many authors considered spectral problems for $p$-Laplace operator; see, for example, [33-38]. These papers contain the results on qualitative properties of the $p$ Laplace spectral problems, convergence of eigenvalue problems, and some estimates for the difference between considered eigenvalues. The applications of our problem do not require the knowledge about the full spectrum of eigenvalues. Therefore we have proved the homogenization theorems only for the first eigenelement of the spectral problem in perforated domain. More precisely, we have proved that the first eigenelement of the spectral problems converges to the corresponding eigenelement of the spectral limit problem. An analogous problem for linear elliptic operators for the twodimensional domain was considered in [15] and for dimension three in [10].

The crucial point in our analysis is the validity of the Friedrichs inequality for functions in perforated domains. We prove this nontrivial result which is of an independent interest. Some papers devoted to this inequality in domains with microinhomogeneous structure are [8-10, 18, 19]. 


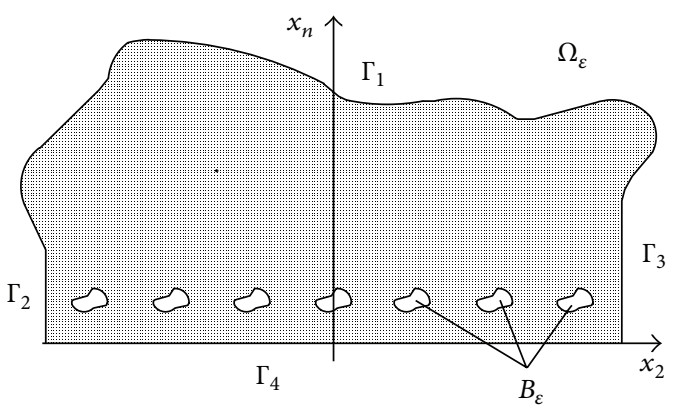

FIGURE 1: Structure of $\Omega_{\varepsilon}$.

\section{Preliminaries and the Main Results}

Let $\Omega \subset \mathbb{R}^{n-1} \times\left\{x_{n}>0\right\}, n>2$, be a domain with boundary $\partial \Omega=\Gamma$. We assume that $\Gamma$ is piece-wise smooth and consists of the parts $\Gamma_{i}, i=1,2,3,4: \Gamma=\Gamma_{1} \cup \Gamma_{2} \cup \Gamma_{3} \cup \Gamma_{4}$, where $\Gamma_{4}=$ $[-1 / 2 ; 1 / 2]^{n-1} \cap\left\{x_{n}=0\right\}, \Gamma_{2}, \Gamma_{3}$ are orthogonal to $\left\{x_{n}=0\right\}$ and belong to the planes $\left\{x_{1}=-1 / 2\right\}$ and $\left\{x_{1}=1 / 2\right\}$ correspondingly, and $\Gamma \backslash \Gamma_{4}$ is a smooth surface. In the sequel $\varepsilon=$ $1 /(2 \mathcal{N}+1)$ is a small parameter, $\mathcal{N} \in \mathbb{N}, \mathcal{N} \gg 1$.

Consider the set $B \subset \mathbb{R}^{n}$ belonging to the ball

$$
K=\left\{\xi: \sum_{i=1}^{n-1} \xi_{i}^{2}+\left(\xi_{n}-\frac{1}{2}\right)^{2}<a^{2}\right\}, \quad 0<a<\frac{1}{2}
$$

and having a smooth boundary. If one multiplies each coordinate of $B$ with parameter $\varepsilon$ and does integer translations of this set along $\Gamma_{4}$, we obtain the set denoted by $B_{\varepsilon}$. Let $\Gamma_{\varepsilon}=\partial B_{\varepsilon}$. Define the perforated domain $\Omega_{\varepsilon}$ as $\Omega \backslash \overline{B_{\varepsilon}}$. See the illustration for cut of $\Omega_{\varepsilon}$ on Figure 1 .

Denote by $W^{1, p}\left(\Omega_{\varepsilon}, \Gamma_{\varepsilon}\right)$ a set of functions from $W^{1, p}\left(\Omega_{\varepsilon}\right)$ with zero trace on $\Gamma_{\varepsilon}$. Analogously, by $W^{1, p}\left(\Omega, \Gamma_{4}\right)$ we define a set of functions from $W^{1, p}(\Omega)$ with zero trace on $\Gamma_{4}$. We also consider the space $C_{0}^{\infty}\left(\bar{\Omega}, \Gamma_{4}\right)$, which is the set of functions from $C^{\infty}(\bar{\Omega})$, vanishing in the neighborhood of $\Gamma_{4}$. Analogously, $C_{0}^{\infty}\left(\bar{\Omega}_{\varepsilon}, \Gamma_{\varepsilon}\right)$ denotes the set of functions from $C^{\infty}\left(\bar{\Omega}_{\varepsilon}\right)$, vanishing in a neighborhood of $\Gamma_{\varepsilon}$.

Remark 1. One can extend the functions $u_{\varepsilon} \in W^{1, p}\left(\Omega_{\varepsilon}, \Gamma_{\varepsilon}\right)$ into $B_{\varepsilon}$ by zero. For the extended function we keep the same notation. It is true that $u_{\varepsilon}$ belongs to $W^{1, p}(\Omega)$; see [24].

Definition 2. For $2 \leq p<n$ define the operator

$$
\Delta u_{p}(x) \equiv \sum_{j=1}^{n} \frac{\partial}{\partial x_{j}}\left(|\nabla u(x)|^{p-2} \frac{\partial u(x)}{\partial x_{j}}\right) .
$$

We consider the following spectral problem:

$$
\begin{aligned}
-\Delta_{p} u_{\varepsilon}(x) & =\lambda_{\varepsilon}\left|u_{\varepsilon}\right|^{p-2} u_{\varepsilon} \quad \text { in } \Omega_{\varepsilon}, \\
u_{\varepsilon} & =0 \quad \text { on } \Gamma_{\varepsilon}, \\
\frac{\partial u_{\varepsilon}}{\partial_{p} \nu} & \equiv|\nabla u|^{p-2}(\nabla u, v)=0 \quad \text { on } \partial \Omega,
\end{aligned}
$$

where $v$ is the unit outward normal vector to the boundary of $\Omega$.

Definition 3. One says that $\lambda_{\varepsilon}$ is an eigenfunction to problem (3) if there exists $u_{\varepsilon} \in W^{1, p}\left(\Omega_{\varepsilon}, \Gamma_{\varepsilon}\right) \backslash\{0\}$, satisfying the integral identity

$$
\begin{aligned}
& \int_{\Omega_{\varepsilon}} \sum_{j=1}^{n}\left|\nabla u_{\varepsilon}(x)\right|^{p-2} \frac{\partial u_{\varepsilon}(x)}{\partial x_{j}} \frac{\partial \varphi_{\varepsilon}(x)}{\partial x_{j}} d x \\
& \quad=\lambda_{\varepsilon} \int_{\Omega_{\varepsilon}}\left|u_{\varepsilon}\right|^{p-2} u_{\varepsilon} \varphi_{\varepsilon} d x
\end{aligned}
$$

for every $\varphi_{\varepsilon} \in W^{1, p}\left(\Omega_{\varepsilon}, \Gamma_{\varepsilon}\right)$. The couple $\left(u_{\varepsilon}, \lambda_{\varepsilon}\right)$ is called the solution to (3).

We will show that the problem

$$
\begin{aligned}
-\Delta_{p} u_{0}(x) & =\lambda_{0}\left|u_{0}\right|^{p-2} u_{0} \quad \text { in } \Omega, \\
u_{0} & =0 \quad \text { on } \Gamma_{4}, \\
\frac{\partial u_{0}}{\partial_{p} \nu} & \equiv\left|\nabla u_{0}\right|^{p-2} \frac{\partial u_{0}}{\partial \nu}=0 \quad \text { on } \partial \Omega \backslash \bar{\Gamma}_{4}
\end{aligned}
$$

is homogenized (the limit one) for (3).

As usual, we understand the solution to this boundaryvalue problem in the weak sense, that is, iff $u_{0} \in W^{1, p}\left(\Omega, \Gamma_{4}\right) \backslash$ $\{0\}$ satisfies

$$
\begin{aligned}
& \int_{\Omega} \sum_{j=1}^{n}\left|\nabla u_{0}(x)\right|^{p-2} \frac{\partial u_{0}(x)}{\partial x_{j}} \frac{\partial \varphi(x)}{\partial x_{j}} d x \\
& \quad=\lambda_{0} \int_{\Omega}\left|u_{0}\right|^{p-2} u_{0} \varphi d x
\end{aligned}
$$

for every $\varphi \in W^{1, p}\left(\Omega, \Gamma_{4}\right)$.

Moreover, we prove the following results.

Theorem 4. Assume that $F \in W^{-1, q}(\Omega), 1 / p+1 / q=1,2 \leq$ $p<\infty, q>1$, and $K$ is an arbitrary compact set belonging to the complex plane $\mathbb{C} ; K$ does not contain the eigenvalues of problem (5). Then the following statements hold:

(1) There exists a number $\varepsilon_{0}>0$, such that the unique solution to the problem

$$
\begin{aligned}
-\Delta_{p} U_{\varepsilon}(x) & =\lambda\left|U_{\varepsilon}\right|^{p-2} U_{\varepsilon}+F \quad \text { in } \Omega_{\varepsilon}, \\
U_{\varepsilon} & =0 \quad \text { on } \Gamma_{\varepsilon}, \\
\frac{\partial U_{\varepsilon}}{\partial_{p} \nu} & \equiv\left|\nabla U_{\varepsilon}\right|^{p-2}\left(\nabla U_{\varepsilon}, \nu\right)=0 \quad \text { on } \partial \Omega
\end{aligned}
$$

does exist for all $\varepsilon<\varepsilon_{0}$ and for all $\lambda \in K$. Moreover, the uniform (in $\varepsilon$ and $\lambda$ ) estimate

$$
\left\|U_{\varepsilon}\right\|_{W^{1, p}} \leq C\|F\|_{L_{q}}
$$

is valid, where $C$ does not depend on $U_{\varepsilon}$ and $F$. 
(2) It yields that

$$
\left\|U_{\varepsilon}-U_{0}\right\|_{W^{1, p}} \longrightarrow 0 \text { when } \varepsilon \longrightarrow 0,
$$

where $U_{0}$ is the unique solution of the problem

$$
\begin{aligned}
-\Delta_{p} U_{0}(x) & =\lambda\left|U_{0}\right|^{p-2} U_{0}+F \quad \text { in } \Omega, \\
U_{0} & =0 \quad \text { on } \Gamma_{4}, \\
\frac{\partial U_{0}}{\partial_{p} \nu} & \equiv\left|\nabla U_{0}\right|^{p-2} \frac{\partial U_{0}}{\partial \nu}=0 \quad \text { on } \partial \Omega \backslash \bar{\Gamma}_{4} .
\end{aligned}
$$

Here the solutions to problems (7) and (10) are understood in the weak sense, that is, iff $U_{\delta}, \delta=\left\{\begin{array}{c}\varepsilon, \\ 0\end{array}\right.$ satisfies the integral identity

$$
\begin{gathered}
\int_{\Omega} \sum_{j=1}^{n}\left|\nabla U_{\delta}(x)\right|^{p-2} \frac{\partial U_{\delta}(x)}{\partial x_{j}} \frac{\partial \varphi(x)}{\partial x_{j}} d x \\
\quad=\lambda \int_{\Omega}\left|U_{\delta}\right|^{p-2} U_{\delta} \varphi d x+\int_{\Omega} F \varphi d x
\end{gathered}
$$

for every $\varphi \in W^{1, p}\left(\Omega, \Gamma_{4}\right)$.

Theorem 5. The spectrum of problems (3) and (5) is nonempty closed set. Let $\lambda_{\varepsilon}^{1}, \lambda_{0}^{1}$ be the first eigenvalues of problems (3) and (5), respectively. Then

$$
\lambda_{\varepsilon}^{1} \longrightarrow \lambda_{0}^{1} \quad \text { as } \varepsilon \longrightarrow 0 .
$$

Moreover, if $u_{\varepsilon}, u_{0}$ are corresponding eigenfunctions, normalized in $L_{p}$, then up to a subsequence,

$$
\left\|u_{\varepsilon}-u_{0}\right\|_{W^{1, p}} \longrightarrow 0
$$

The proofs of analogous theorems for linear boundaryvalue problems were given in $[6,12-15]$ for different types of singular perturbations. The following lemma, which is proved in Section 3.1, is necessary for our analysis.

Lemma 6. Let $v_{\varepsilon}$ be a sequence of functions from $W^{1, p}\left(\Omega_{\varepsilon}, \Gamma_{\varepsilon}\right)$ and assume that $v_{\varepsilon} \rightarrow v^{*}$ weakly in $W^{1, p}(\Omega)$ when $\varepsilon \rightarrow 0$. Then $v^{*} \in W^{1, p}\left(\Omega, \Gamma_{4}\right)$.

For the questions on existence of solutions to the discussed problems we will refer to the following general result (see [39]).

Theorem 7. Let $V$ be reflexive separable Banach space. Assume that the operator $A: V \rightarrow V^{\prime}$ has the following properties:

(i) $A$ is bounded and semicontinuous: $\|A\|<\infty, \forall u$, $v, w \in V$; the function $s \rightarrow(A(u+s v), w)$ is continuous as a function mapping $\mathbb{R}$ into $\mathbb{R}$.

(ii) $A$ is monotone: $(A(u)-A(v), u-v) \geq 0 \quad \forall u, v \in V$.

(iii) Consider

$$
\frac{(A(u), u)}{\|u\|} \longrightarrow+\infty \quad \text { as }\|u\| \longrightarrow+\infty
$$

Then for any $f \in V^{\prime}$ there exists $u \in V$ such that $A(u)=f$.
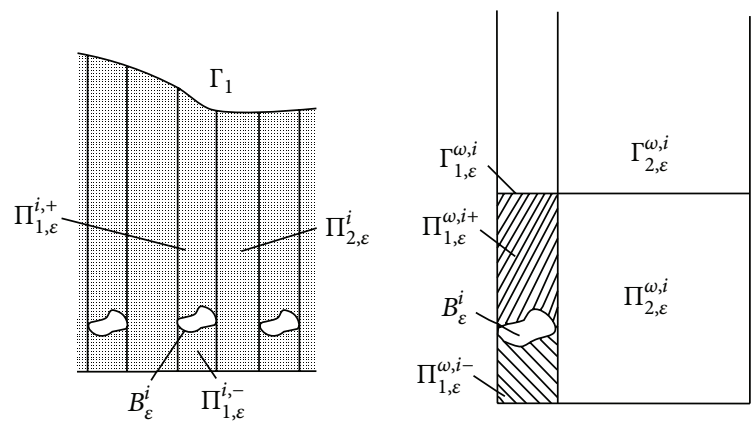

FIgURE 2: Parts of $\Omega_{\varepsilon}$.

\section{The Friedrichs Inequality}

In our analysis we will need the Friedrichs inequality for functions $v_{\varepsilon} \in W^{1, p}\left(\Omega_{\varepsilon}, \Gamma_{\varepsilon}\right)$. We prove this result.

Theorem 8. The inequality

$$
\int_{\Omega_{\varepsilon}}\left|v_{\varepsilon}\right|^{p} d x \leq K \int_{\Omega_{\varepsilon}}\left|\nabla v_{\varepsilon}\right|^{p} d x
$$

holds for any functions $v_{\varepsilon} \in W^{1, p}\left(\Omega_{\varepsilon}, \Gamma_{\varepsilon}\right)$, where the constant $K$ does not depend on $\varepsilon$.

Proof. To demonstrate the technique of proving and avoiding the heavy $n$-dimensional notations we assume that $n=2$. The case of arbitrary $n>2$ can be done by repeating all lines of the present proof.

Let the length of projection of $B$ on axis $\xi_{1}$ equal $b$. Denote

$$
B_{\varepsilon}^{i}=\left\{x \in \Omega: \varepsilon^{-1}\left(x_{1}-i, x_{2}\right) \in B\right\}, \quad i \in \mathbb{Z} .
$$

Represent the domain $\Omega_{\varepsilon}$ as follows:

$$
\Omega_{\varepsilon}=\bigcup_{i=-\mathcal{N}}^{\mathcal{N}}\left(\Pi_{1, \varepsilon}^{i+} \cup \Pi_{1, \varepsilon}^{i-}\right) \cup \bigcup_{i=-\mathcal{N}-1}^{\mathcal{N}} \Pi_{2, \varepsilon}^{i} \cup R_{\varepsilon}
$$

where $\Pi_{1, \varepsilon}^{i+}$ is the domain (a part of the vertical strip) of the length $b \varepsilon$, bounded from below by the upper part of the boundary $B_{\varepsilon}^{i}$ and bounded from above by a part of the boundary $\Gamma_{1}$; the domain $\Pi_{1, \varepsilon}^{i-}$ is the strip of the length $b \varepsilon$, bounded from above by the lower part of $B_{\varepsilon}^{i}$ and bounded from below by the line $x_{2}=0 ; \Pi_{2, \varepsilon}^{i}, i=-\mathcal{N}, \ldots, \mathcal{N}-1$, is the strip of the length $(1-b) \varepsilon$, bounded from below by the line $x_{2}=0$ and bounded from above by a part of $\Gamma_{1}$ and having the common vertical bounds with $\Pi_{1, \varepsilon}^{i+}, \Pi_{1, \varepsilon}^{i+1+}$ (see Figure 2); the domains $\Pi_{2, \varepsilon}^{-\mathcal{N}-1}$ and $\Pi_{2, \varepsilon}^{\mathcal{N}}$ are vertical strips bounded from below by $x_{2}=0$ and bounded from above by a part of $\Gamma_{1}$ and having the common vertical bounds with $\Pi_{1, \mathcal{E}}^{-\mathcal{N}+}$ and $\Pi_{1, \mathcal{E}}^{\mathcal{N}+}$, correspondingly. The sum of widths for $\Pi_{2, \varepsilon}^{-\mathcal{N}-1}$ and $\Pi_{2, \varepsilon}^{\mathcal{N}}$ is $(1-$ b) $\varepsilon$. Finally, $R_{\varepsilon}$ is the remaining part of $\Omega_{\varepsilon}$ having the bound belonging to $\Gamma_{1}$.

Let $\omega>\varepsilon$. Define by $\Pi_{1, \varepsilon}^{\omega, i+}$ a domain $\Pi_{1, \varepsilon}^{i+} \bigcap\left\{x_{2}<\omega\right\}$ (see Figure 2). Moreover, let $\Gamma_{1, \varepsilon}^{\omega, i}$ be the segment of the line $x_{n}=\omega$, 


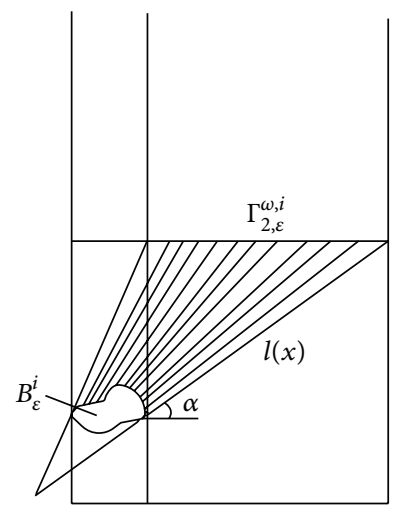

Figure 3: Tangential lines.

which is upper bound for $\Pi_{1, \varepsilon}^{\omega, i+}$. Analogously we define the sets $\Gamma_{2, \varepsilon}^{\omega, i}$ and $\Pi_{2, \varepsilon}^{\omega, i}$. Without loss of generality we can assume that $v_{\varepsilon}$ is real-valued function. Denote by $C_{0}^{1}\left(\bar{\Omega}_{\varepsilon}, \Gamma_{\varepsilon}\right)$ functions from $C^{1}\left(\bar{\Omega}_{\varepsilon}\right)$, vanishing in the neighborhood of $\Gamma_{\varepsilon}$. Assume first that $v_{\varepsilon} \in C_{0}^{1}\left(\bar{\Omega}_{\varepsilon}, \Gamma_{\varepsilon}\right)$. Let $\left(x_{1}, \omega\right) \in \Pi_{1, \varepsilon}^{i+}$, and point $\left(x_{1}, \widetilde{x}_{2}\right)$ belongs to the bottom of $\Pi_{1, \varepsilon}^{i+}$. Since $v_{\varepsilon}\left(x_{1}, \widetilde{x}_{2}\right)=0$, then the Newton-Leibnitz formula gives

$$
v_{\varepsilon}\left(x_{1}, \omega\right)=\int_{\tilde{x}_{2}}^{\omega} \frac{\partial v_{\varepsilon}}{\partial x_{2}} d x_{2} .
$$

Taking the power $p$ on both sides of that equality and using the Hölder inequality, we have

$$
\begin{aligned}
\left|v_{\varepsilon}\left(x_{1}, \omega\right)\right|^{p} & =\left|\int_{\tilde{x}_{2}}^{\omega} \frac{\partial v_{\varepsilon}}{\partial x_{2}} d x_{2}\right|^{p} \leq \omega^{p / q} \int_{\tilde{x}_{2}}^{\omega}\left|\frac{\partial v_{\varepsilon}}{\partial x_{2}}\right|^{p} d x_{2} \\
& \leq \omega^{p / q} \int_{\tilde{x}_{2}}^{\omega}\left|\nabla v_{\varepsilon}\right|^{p} d x_{2} .
\end{aligned}
$$

Integrate both sides of that inequality over $\Gamma_{1, \varepsilon}^{\omega, i}$ with respect to $x_{1}$ and assume that the function $v_{\varepsilon}$ is extended by zero into $B_{\varepsilon}^{i} \cup \Pi_{1, \varepsilon}^{i-}$. One gets

$$
\int_{\Gamma_{1, \varepsilon}^{(\omega, j}}\left|v_{\varepsilon}\left(x_{1}, \omega\right)\right|^{p} d x_{1} \leq \omega^{p / q} \int_{\Pi_{1, \varepsilon}^{\omega, i,}}\left|\nabla v_{\varepsilon}\right|^{p} d x .
$$

Consider now the rectangle $\Pi_{2, \varepsilon}^{i}, i=-\mathcal{N}, \ldots, \mathcal{N}$, which touches $B_{\varepsilon}^{i}$ from the right. Draw the tangential lines to $B_{\varepsilon}^{i}$ from both ends of segment $\Gamma_{2, \varepsilon}^{\omega, i}$ (see Figure 3).

It is easy to see that the angle $\alpha$ between the tangential lines and $O x_{1}$ belongs to $\left(\alpha_{0}, \pi / 2\right)$, where $\alpha_{0}>0$ does not depend on $\varepsilon$ and $\omega$. We remember that our assumption was $\omega>\varepsilon$. This follows from the fact that the diameter of the set and the distance between them are of the same order. Connect all points of $\Gamma_{2, \varepsilon}^{\omega, i}$ with boundary of $B_{\varepsilon}^{i}$ such that the intersection of these lines coincides with the intersection point of tangents. Thus, we have a beam of lines with directors $l(x)$. The angle between each line and $O x_{1}$ belongs to $\left(\alpha_{0}, \pi / 2\right)$.
Let $\left(x_{1}, \omega\right) \in \Gamma_{2, \varepsilon}^{\omega, i}$, and $\left(\tilde{x}_{1}, \tilde{x}_{2}\right) \in \partial B_{\varepsilon}^{i} \cap l\left(x_{1}, \omega\right)$. Since $v_{\varepsilon}\left(\widetilde{x}_{1}\right.$, $\left.\tilde{x}_{2}\right)=0$, then

$$
v_{\varepsilon}\left(x_{1}, \omega\right)=\int_{\left(\tilde{x}_{1}, \tilde{x}_{2}\right)}^{\left(x_{1}, \omega\right)} \frac{\partial v_{\varepsilon}}{\partial l} d l .
$$

Analogously to (19), taking the power $p$ on both sides of that formula and using the Hölder inequality, one obtains

$$
\begin{aligned}
\left|v_{\varepsilon}\left(x_{1}, \omega\right)\right|^{p} & \leq\left|\frac{\omega}{\sin \alpha_{0}}\right|^{p / q} \int_{\left(\tilde{x}_{1}, \tilde{x}_{2}\right)}^{\left(x_{1}, \omega\right)}\left|\frac{\partial v_{\varepsilon}}{\partial l}\right|^{p} d l \\
& \leq\left|\frac{\omega}{\sin \alpha_{0}}\right|^{p / q} \int_{\left(\widetilde{x}_{1}, \tilde{x}_{2}\right)}^{\left(x_{1}, \omega\right)}\left|\nabla v_{\varepsilon}\right|^{p} d l .
\end{aligned}
$$

Integrating both sides of that inequality over $\Gamma_{2, \varepsilon}^{\omega, i}$ with respect to $x_{1}$ and replacing the right-hand side by the greater integral, we get

$$
\begin{aligned}
& \int_{\Gamma_{2, \varepsilon}^{\omega, i}}\left|v_{\varepsilon}\left(x_{1}, \omega\right)\right|^{p} d x_{1} \\
& \leq\left|\frac{\omega}{\sin \alpha_{0}}\right|^{p / q} \int_{\Pi_{2, \varepsilon}^{\omega, i} \cup \Pi_{1, \varepsilon}^{\omega, j i} \cup \Pi_{1, \varepsilon}^{\omega, i-}}\left|\nabla v_{\varepsilon}\right|^{p} d x, \\
& i=-\mathcal{N}, \ldots, \mathcal{N} .
\end{aligned}
$$

It remains to estimate the integral over $\Gamma_{2, \varepsilon}^{\omega,-\mathcal{N}-1}$. We use the same technique as for $\Gamma_{2, \varepsilon}^{\omega, i}, i=-\mathcal{N}, \ldots, \mathcal{N}$. In this case one needs to consider the domain $B_{\varepsilon}^{-\mathcal{N}}$ which is bordered with the strip $\Pi_{2, \varepsilon}^{-\mathcal{N}-1}$ from the right. Analogously, we get

$$
\begin{aligned}
& \int_{\Gamma_{2, \varepsilon}^{(\omega,-\mathcal{N}-1}}\left|v_{\varepsilon}\left(x_{1}, \omega\right)\right|^{p} d x_{1} \\
& \quad \leq C \omega^{p / q} \int_{\Pi_{2, \varepsilon}^{\omega,-\mathcal{N}-1} \cup \Pi_{1, \varepsilon}^{\omega,-\mathcal{N}} \cup \Pi_{1, \varepsilon}^{\omega,-\mathcal{N}-}}\left|\nabla v_{\varepsilon}\right|^{p} d x .
\end{aligned}
$$

Let $\Gamma_{4}^{\omega}=\bar{\Omega} \cap\left\{x_{2}=\omega\right\}$ be the segment connecting the points $(-1 / 2, \omega)$ and $(1 / 2, \omega)$; it means that $\Gamma_{4}^{\omega}=\bigcup_{i} \overline{\Gamma_{1, \varepsilon}^{\omega, i}} \cup \overline{\Gamma_{2, \varepsilon}^{\omega, i}}$. Summing up inequalities (20), (23), and (24), one gets

$$
\int_{\Gamma_{4}^{\omega}}\left|v_{\varepsilon}(x)\right|^{p} d x_{1} \leq C_{1} \omega^{p / q} \int_{\Omega_{\varepsilon}}\left|\nabla v_{\varepsilon}\right|^{p} d x .
$$

Finally, integrating (25) with respect to $\omega$, we obtain that

$$
\int_{\Omega_{\varepsilon}}\left|v_{\varepsilon}(x)\right|^{p} d x_{1} d x_{2} \leq K \int_{\Omega_{\varepsilon}}\left|\nabla v_{\varepsilon}\right|^{p} d x .
$$

Approximating the functions from $W^{1, p}\left(\Omega_{\varepsilon}, \Gamma_{\varepsilon}\right)$ by smooth functions, we conclude that inequality (26) is valid for $v_{\varepsilon} \in W^{1, p}\left(\Omega_{\varepsilon}, \Gamma_{\varepsilon}\right)$. The proof is complete.

Remark 9. (1) Extending functions from $W^{1, p}\left(\Omega_{\varepsilon}, \Gamma_{\varepsilon}\right)$ by zero into $\bigcup_{i} B_{\varepsilon}^{i}$ we obtain the Friedrichs inequality in $\Omega$ :

$$
\left\|v_{\varepsilon}\right\|_{L_{p}(\Omega)}^{p} \leq K\left\|\nabla v_{\varepsilon}\right\|_{L_{p}(\Omega)}^{p} \quad \text { for } v_{\varepsilon} \in W^{1, p}\left(\Omega_{\varepsilon}, \Gamma_{\varepsilon}\right) .
$$


(2) The validity of Friedrichs inequality means that one can introduce in $W^{1, p}\left(\Omega_{\varepsilon}, \Gamma_{\varepsilon}\right)$ the norm $\|v\|_{W^{1, p}} \equiv\|\nabla v\|_{L_{p}}$ which is equivalent to

$$
\|v\|_{W^{1, p}} \equiv\|v\|_{L_{p}}+\|\nabla v\|_{L_{p}}
$$

\subsection{Proof of Lemma 6}

Proof. First we point out that with the same method of proof inequality (25) is valid also for the case of an arbitrary $n>2$, where

$$
\Gamma_{4}^{\omega}=\bar{\Omega} \cap\left\{x_{n}=\omega\right\}
$$

Approximating the functions from $W^{1, p}\left(\Omega_{\varepsilon}, \Gamma_{\varepsilon}\right)$ by smooth functions, we conclude that inequality (25) is valid for $v_{\varepsilon} \in$ $W^{1, p}\left(\Omega_{\varepsilon}, \Gamma_{\varepsilon}\right)$. Using (25) and keeping in mind the uniform boundedness of the sequence $v_{\varepsilon}$, we obtain that

$$
\int_{\Gamma_{4}^{\omega}}\left|v_{\varepsilon}\left(x_{1}, \ldots, x_{n-1}, \omega\right)\right|^{p} d x \leq \omega^{p / q} C
$$

Now, we can pass to the limit in (30) when $\varepsilon \rightarrow 0$ and find that

$$
\int_{\Gamma_{4}^{\omega}}\left|v^{*}\right|^{p} d x \leq \omega^{p / q} C_{3} .
$$

Due to the fact that $\omega$ is an arbitrary small positive number and $v^{*} \in W^{1, p}(\Omega)$, it follows from (31) that $v^{*}=0$ on $\Gamma_{4}$. The proof is complete.

\section{Proof of Theorem 4}

For the proof we need the following lemma.

Lemma 10. Let $Q$ be an arbitrary compact set in the complex plane and $\lambda \in Q$. Suppose that the estimate

$$
\left\|U_{\varepsilon}\right\|_{p} \leq C\|F\|_{q}
$$

holds uniformly in $\varepsilon$ and $\lambda$ for any solution $U_{\varepsilon}$ of the boundaryvalue problem (7), which is normalized in $L_{p}(\Omega)$. Then estimate (32) holds also for any solution of problem (7) when $\lambda \in Q$.

Proof. Let us remember first that we denote by $\|\cdot\|_{p}$ the norm in $L_{p}$ space. If $\left\|U_{\varepsilon}\right\|_{p} \neq 1$, then by setting $V_{\varepsilon}=U_{\varepsilon} /\left\|U_{\varepsilon}\right\|_{p}$, we obtain that

$$
\left\|V_{\varepsilon}\right\|_{p}=1
$$

and the function $V_{\varepsilon}$ satisfies the identity

$$
\begin{aligned}
& \int_{\Omega} \sum_{j=1}^{n}\left|\nabla V_{\varepsilon}(x)\right|^{p-2} \frac{\partial V_{\varepsilon}(x)}{\partial x_{j}} \frac{\partial \varphi(x)}{\partial x_{j}} d x \\
& \quad=\lambda \int_{\Omega}\left|V_{\varepsilon}\right|^{p-2} V_{\varepsilon} \varphi d x+\int_{\Omega} F_{\varepsilon} \varphi d x,
\end{aligned}
$$

where

$$
F_{\varepsilon}=\frac{F}{\left\|U_{\varepsilon}\right\|_{p}^{p-1}} .
$$

Hence, due to the assumptions we see that the estimate

$$
\left\|V_{\varepsilon}\right\|_{p} \leq C\left\|F_{\varepsilon}\right\|_{q}
$$

holds for $V_{\varepsilon}$. Multiplying the last inequality by $\left\|U_{\varepsilon}\right\|_{p}$ and using (33) and (35), we obtain the estimate (32) (probably with a different constant) for any $U_{\varepsilon}$. The proof is complete.

\subsection{Proof of Part 1}

Step 1. The existence of the solution to problem (7) can be proved with help of Theorem 7. Indeed, we take $V=$ $W^{1, p}\left(\Omega, \Gamma_{\varepsilon}\right)$. Introduce the operator $A(u)=-\sum_{i}\left(\partial / \partial x_{i}\right)(|\partial u|$ $\left.\left.\partial x_{i}\right|^{p-2}\left(\partial u / \partial x_{i}\right)\right)$. For $u, v \in W^{1, p}\left(\Omega, \Gamma_{\varepsilon}\right)$ we define

$$
\begin{aligned}
(A(u), v) & =-\int_{\Omega} \sum_{i} \frac{\partial}{\partial x_{i}}\left(\left|\frac{\partial u}{\partial x_{i}}\right|^{p-2} \frac{\partial u}{\partial x_{i}}\right) v d x \\
& =\int_{\Omega} \sum_{i}\left|\frac{\partial u}{\partial x_{i}}\right|^{p-2} \frac{\partial u}{\partial x_{i}} \frac{\partial v}{\partial x_{i}} d x .
\end{aligned}
$$

Moreover, for $u \in W^{1, p}\left(\Omega, \Gamma_{\varepsilon}\right)$ it holds that $\lambda|u|^{p-1} u \in L_{q}(\Omega$, $\left.\Gamma_{\varepsilon}\right)$, and for $F \in L_{q}(\Omega)$ we can introduce the functional on $W^{1, p}\left(\Omega, \Gamma_{\varepsilon}\right)$ :

$$
\left(\lambda|u|^{p-1} u+F, v\right)=\int_{\Omega} \lambda|u|^{p-1} u v d x+\int_{\Omega} F v d x
$$

It is clear that $U_{\varepsilon}$ is the solution to (7) iff $\left(A\left(U_{\varepsilon}\right), v\right)=$ $\left(\lambda\left|U_{\varepsilon}\right|^{p-1} U_{\varepsilon}+F, v\right)$ for any $v \in W^{1, p}\left(\Omega, \Gamma_{\varepsilon}\right)$. Let us verify the properties of operator $A$. We take $u \in W^{1, p}$ with $\|u\|_{W^{1, p}}=1$. Then

$$
\|A(u)\|_{W^{-1, q}}=(A(u), u)=\|\nabla u\|_{L_{p}}^{p} \leq C\|u\|_{W^{1, p}}^{p} \leq C .
$$

One can check the semicontinuity and monotonicity of operator $A$ either directly or by using the following general result (see [39]).

Proposition 11. If convex functional $v \rightarrow J(v)$ is differentiable in Gato sense, that is, there exists such continuous linear mapping $v \rightarrow J^{\prime}(u) \cdot v$ of space $V$ onto $\mathbb{R}$ such that

$$
\lim _{s \rightarrow 0} \frac{1}{s}(J(u+s v)-J(u))=J^{\prime} \cdot v,
$$

then the mapping $u \rightarrow J^{\prime}(u)$ is monotone and semicontinuous.

Let us define the functional

$$
J(v)=\frac{1}{p} \sum_{i=1}^{2} \int_{\Omega}\left|\frac{\partial v}{\partial x_{i}}\right|^{p} d x
$$


on the space $V=W^{1, p}\left(\Omega, \Gamma_{\varepsilon}\right)$. It is easy to check that $J$ is convex and differentiable in Gato sense and $J^{\prime}(u)=A(u)$. Hence, $A$ is semicontinuous and monotone operator. Finally, taking into account Remark 9 we calculate that

$$
\begin{array}{r}
\frac{(A(u), u)}{\|u\|}=\frac{\|\nabla u\|_{L_{p}}^{p}}{\|u\|_{W^{1, p}}}=C\|u\|_{W^{1, p}}^{p-1} \\
\text { as }\|u\|_{W^{1, p}} \longrightarrow+\infty .
\end{array}
$$

Therefore by Theorem 7 for any $F \in L_{q}$ there exists a function $U_{\varepsilon}$ satisfying $A\left(U_{\varepsilon}\right)=\lambda\left|U_{\varepsilon}\right|^{p-2}+F$.

Step 2. Let us derive now estimate (8). Substitute $\varphi$ with $U_{\varepsilon}$ in (11). Then it follows that

$$
\begin{aligned}
\int_{\Omega}\left|\nabla U_{\varepsilon}(x)\right|^{p} d x & =\lambda \int_{\Omega}\left|U_{\varepsilon}\right|^{p} d x+\int_{\Omega} F U_{\varepsilon} d x, \\
\left\|U_{\varepsilon}\right\|_{W^{1, p}}^{p} & =(\lambda+1) \int_{\Omega}\left|U_{\varepsilon}\right|^{p} d x+\int_{\Omega} F U_{\varepsilon} d x \\
& \leq|\lambda+1|\left\|U_{\varepsilon}\right\|_{L_{p}}^{p}+\|F\|_{L_{q}}\left\|U_{\varepsilon}\right\|_{L_{p}} \\
& \leq C\left\|U_{\varepsilon}\right\|_{W^{1, p}}\left(\left\|U_{\varepsilon}\right\|_{L_{p}}^{p-1}+\|F\|_{L_{p}}\right) .
\end{aligned}
$$

Hence,

$$
\left\|U_{\varepsilon}\right\|_{W^{1, p}}^{p-1} \leq C\left(\left\|U_{\varepsilon}\right\|_{L_{p}}^{p-1}+\|F\|_{L_{q}}\right) .
$$

Assume now that estimate (8) does not hold, that is, that there exists a sequence $\left\{\varepsilon_{k}\right\} \varepsilon_{k} \rightarrow 0$ when $k \rightarrow \infty, F_{k} \in L_{q}(\Omega)$ and $\lambda_{k}$ such that the inequality

$$
\left\|U_{\varepsilon_{k}}\right\|_{W^{1, p}}>k\left\|F_{k}\right\|_{q}
$$

holds for the solutions to problem (7), where

$$
\begin{aligned}
& \varepsilon=\varepsilon_{k}, \\
& \lambda=\lambda_{k}, \\
& F=F_{k} .
\end{aligned}
$$

Due to Lemma 10 we may assume without loss of generality that the sequence $\left\{U_{\varepsilon}\right\}$ is normalized in $L_{p}(\Omega)$, that is, that

$$
\left\|U_{\varepsilon_{k}}\right\|_{p}=1
$$

Then, by using (44) and (45), we obtain that

$$
\begin{array}{r}
\left\|U_{\varepsilon_{k}}\right\|_{W^{1, p}} \leq C, \\
\left\|F_{k}\right\|_{q}<\frac{C}{k},
\end{array}
$$

when $k \rightarrow \infty$.

Hence, there exists a subsequence $\left\{k^{\prime}\right\}$ of indexes $\{k\}$ and $U^{*} \in W^{1, p}(\Omega), \lambda^{*}$ such that

$$
\begin{aligned}
& \lambda_{k^{\prime}} \longrightarrow \lambda^{*} \in K, \\
& U_{\varepsilon_{k^{\prime}}} \rightarrow U^{*} \text { in } W^{1, p}(\Omega) \text { when } k^{\prime} \longrightarrow+\infty .
\end{aligned}
$$

By (47) and (49) we have that

$$
U^{*} \neq 0
$$

Moreover, since the operator $A$ is bounded, it follows that $\left\|A\left(U_{\varepsilon_{k^{\prime}}}\right)\right\|_{W^{-1, q}} \leq C$. Therefore we conclude the existence of $\chi \in W^{-1, q}$ such that $A\left(U_{\varepsilon_{k^{\prime}}}\right) \rightarrow \chi$ weakly in $W^{-1, q}$. Suppose that $v$ is an arbitrary fixed function from $C_{0}^{\infty}\left(\bar{\Omega}, \Gamma_{\varepsilon}\right)$. Then $v \in$ $W^{1, p}\left(\Omega, \Gamma_{\varepsilon}\right)$ for all small $\varepsilon$. Substitute in the integral identity (11) $v$ as a test function; $U=U_{\varepsilon_{k^{\prime}}}, \lambda=\lambda_{k^{\prime}}$, and $F=F_{k^{\prime}}$, when $\varepsilon=\varepsilon_{k^{\prime}}$. We have that

$$
\begin{aligned}
& \int_{\Omega} \sum_{j=1}^{n}\left|\nabla U_{\varepsilon_{k^{\prime}}}(x)\right|^{p-2} \frac{\partial U_{\varepsilon_{k^{\prime}}}(x)}{\partial x_{j}} \frac{\partial v(x)}{\partial x_{j}} d x \\
& =\lambda_{k^{\prime}} \int_{\Omega}\left|U_{\varepsilon_{k^{\prime}}}\right|^{p-2} U_{\varepsilon_{k^{\prime}}} v d x+\int_{\Omega} F_{k^{\prime}} v d x .
\end{aligned}
$$

Now we want to pass to the limit as $\varepsilon_{k^{\prime}} \rightarrow 0$. Due to the definition of $A(\cdot)$, we see that

$$
(\chi, v)=\lim _{\varepsilon_{k^{\prime}} \rightarrow 0}\left(\lambda_{k^{\prime}}\left|U_{\varepsilon_{k^{\prime}}}\right|^{p-2} U_{\varepsilon_{k^{\prime}}}+F_{k^{\prime}}, v\right) .
$$

From the other hand,

$$
\begin{aligned}
\left(A\left(U_{\varepsilon_{k^{\prime}}}\right), U_{\varepsilon_{k^{\prime}}}\right) & \longrightarrow\left(\chi, U^{*}\right), \\
\left(\left|U_{\varepsilon_{k^{\prime}}}\right|^{p-2} U_{\varepsilon_{k^{\prime}}}, U_{\varepsilon_{k^{\prime}}}\right) & \longrightarrow\left(\left|U^{*}\right|^{p-2} U^{*}, U^{*}\right) .
\end{aligned}
$$

Let us show now that $\chi=A\left(U^{*}\right)$. The monotonicity of operator $A$ implies

$$
\left(A\left(U_{\varepsilon_{k^{\prime}}}\right)-A(U), U_{\varepsilon_{k^{\prime}}}-U\right) \geq 0
$$

$$
\forall U \in W^{1, p}\left(\Omega, \Gamma_{4}\right) .
$$

Passing to the limit, it yields that

$$
\left(\chi-A(U), U^{*}-U\right) \geq 0 \quad \forall U \in W^{1, p}(\Omega) .
$$

If we take $U=U^{*}-s W, s>0, W \in W^{1, p}\left(\Omega, \Gamma_{4}\right)$, we get

$$
\begin{aligned}
& s\left(\chi-A\left(U^{*}-s W\right), W\right) \geq 0 \\
& \quad \Longrightarrow\left(\chi-A\left(U^{*}-s W\right), W\right) \geq 0 .
\end{aligned}
$$

Let $s \rightarrow 0$. Then

$$
\left(\chi-A\left(U^{*}-s W\right), W\right) \geq 0 \quad \forall U \in W^{1, p}\left(\Omega, \Gamma_{4}\right) .
$$

This exactly means that $\chi=A\left(U^{*}\right)$. Thus, passing to the limit in the integral identity (51) as $\varepsilon_{k^{\prime}} \rightarrow 0$, using (48), (49), and Lemma 6 , we obtain that

$$
\begin{aligned}
\int_{\Omega}\left|\nabla U^{*}(x)\right|^{p-2} \nabla U^{*}(x) \nabla v(x) d x \\
=\lambda^{*} \int_{\Omega}\left|U^{*}\right|^{p-2} U^{*} v d x \quad \text { for } v \in C^{\infty}\left(\Omega, \Gamma_{4}\right) .
\end{aligned}
$$


From the density of the embedding $C^{\infty}\left(\bar{\Omega}, \Gamma_{\varepsilon}\right)$ into $W^{1, p}(\Omega$, $\Gamma_{4}$ ) we can conclude that this inequality holds also for $v \in$ $W^{1, p}\left(\Omega, \Gamma_{4}\right)$. It follows from Lemma 6 that $U^{*} \in W^{1, p}\left(\Omega, \Gamma_{4}\right)$. Due to the fact that $U^{*} \neq 0$ and $v$ is an arbitrary function from $W^{1, p}\left(\Omega, \Gamma_{4}\right)$, it follows that $\lambda^{*} \in K$ is the eigenvalue of the limit problem (5). But we assumed that $K$ did not contain the eigenvalues of the limit problem (5). This contradiction proves estimate (8).

Step 3. Let us prove now the uniqueness of the solution of (7). Let $U_{\varepsilon}^{1}$ and $U_{\varepsilon}^{2}$ be two different solutions. Then

$$
\begin{aligned}
U_{\varepsilon}^{1}-U_{\varepsilon}^{1} & =0 \quad \text { on } \Gamma_{\varepsilon}, \\
\left|\nabla U_{\varepsilon}^{1}\right|^{p-2} \frac{\partial U_{\varepsilon}^{1}}{\partial \nu}-\left|\nabla U_{\varepsilon}^{2}\right|^{p-2} \frac{\partial U_{\varepsilon}^{2}}{\partial \nu}=0 & \text { on } \partial \Omega .
\end{aligned}
$$

This together with equation

$$
\begin{aligned}
& \left(A\left(U_{\varepsilon}^{1}\right)-A\left(U_{\varepsilon}^{2}\right), U_{\varepsilon}^{1}-U_{\varepsilon}^{2}\right)=0 \\
& \quad=\sum_{i} \int_{\Omega}\left(\left|\frac{\partial U_{\varepsilon}^{1}}{\partial x_{i}}\right|^{p-2} \frac{\partial U_{\varepsilon}^{1}}{\partial x_{i}}-\left|\frac{\partial U_{\varepsilon}^{2}}{\partial x_{i}}\right|^{p-2} \frac{\partial U_{\varepsilon}^{2}}{\partial x_{i}}\right) \\
& \cdot\left(\frac{\partial U_{\varepsilon}^{1}}{\partial x_{i}}-\frac{\partial U_{\varepsilon}^{2}}{\partial x_{i}}\right) d x
\end{aligned}
$$

implies that

$$
\frac{\partial U_{\varepsilon}^{1}}{\partial x_{i}}=\frac{\partial U_{\varepsilon}^{2}}{\partial x_{i}} \Longrightarrow U_{\varepsilon}^{1}=U_{\varepsilon}^{2}
$$

due to the boundary conditions. The uniqueness of the solution to (10) can be proved identically.

4.2. Proof of Part 2. Let $\lambda \in K$ be an arbitrary fixed number and assume that the sequence $\left\{\varepsilon_{k}\right\} \rightarrow 0$ when $k \rightarrow+\infty$. Thinking analogously as in Part 1 of the proof, we have existence of a subsequence $\left\{k^{\prime}\right\}$ and a function $U^{*}$ such that $U_{\varepsilon_{k^{\prime}}} \rightarrow U^{*}$ in $W^{1, p}\left(\Omega, \Gamma_{4}\right)$ (and strongly in $L_{p}(\Omega)$ ) when $k^{\prime} \rightarrow+\infty$. In addition, $A\left(U_{\varepsilon_{k^{\prime}}}\right) \rightarrow \chi$ weakly in $W^{-1, q}$. It can be shown exactly in the same way that $\chi=A\left(U^{*}\right)$.

Now by using Lemma 6 we obtain that the limit function $U^{*} \in W^{1, p}\left(\Omega, \Gamma_{4}\right)$. Passing to the limit in the integral identity (51) by means of the same reasoning as in the proof of Part 1 of the theorem, we get that

$$
\begin{aligned}
& \int_{\Omega}\left|\nabla U^{*}(x)\right|^{p-2} \nabla U^{*}(x) \nabla v(x) d x \\
& =\lambda \int\left|U^{*}\right|^{p-2} U^{*} v d x+\int_{\Omega} F v d x,
\end{aligned}
$$

which coincides with the integral identity of problem (10). Since the solution to problem (10) is unique we conclude that $U^{*}=U_{0}$. In addition, from (49) we find that

$$
\begin{gathered}
U_{\varepsilon_{k}} \longrightarrow U_{0} \text { strongly in } L_{p}(\Omega) \\
\text { and weakly in } W^{1, p}(\Omega),
\end{gathered}
$$

$$
A\left(U_{\varepsilon_{k}}\right) \rightarrow A\left(U_{0}\right) \text { in } W^{-1, q} \text { when } \varepsilon \longrightarrow 0 .
$$

From the integral identities we have that

$$
\begin{aligned}
\int_{\Omega} & \left(\left|\nabla U_{\varepsilon_{k}}\right|^{p-2} \nabla U_{\varepsilon_{k}}-\left|\nabla U_{0}\right|^{p-2} \nabla U_{0}\right) \\
& \cdot \nabla\left(U_{\varepsilon_{k}}-U_{0}\right) d x \\
= & \lambda \int_{\Omega}\left|U_{\varepsilon_{k}}\right|^{p-2} U_{\varepsilon_{k}} U_{0} d x \\
& -\int_{\Omega}\left|\nabla U_{0}\right|^{p-2} \nabla U_{0}\left(\nabla U_{\varepsilon_{k}}-\nabla U_{0}\right) d x \\
& +\int_{\Omega} F\left(U_{\varepsilon_{k}}-U_{0}\right) d x .
\end{aligned}
$$

Due to convergences (63) the right-hand side tends to zero in the limit; therefore

$$
\begin{gathered}
\lim _{k \rightarrow \infty} \int_{\Omega}\left(\left|\nabla U_{\varepsilon_{k}}\right|^{p-2} \nabla U_{\varepsilon_{k}}-\left|\nabla U_{0}\right|^{p-2} \nabla U_{0}\right) \\
\cdot \nabla\left(U_{\varepsilon_{k}}-U_{0}\right) d x=0 .
\end{gathered}
$$

By using the inequality

$$
2^{1-p}|a-b|^{p} \leq\left(|a|^{p-2} a-|b|^{p-2} b\right) \cdot(a-b),
$$

$$
p \geq 2 \text {, }
$$

with $a=\nabla U_{\varepsilon_{k}}$ and $b=\nabla U_{0}$ we can show the strong convergence of gradients:

$$
\lim _{k \rightarrow \infty} \int_{\Omega}\left|\nabla U_{\varepsilon_{k}}-\nabla U_{0}\right|^{p} d x=0
$$

Thus, we have proved that, up to a subsequence,

$$
\left\|U_{\varepsilon}-U_{0}\right\|_{W^{1, p}} \longrightarrow 0 .
$$

The proof is complete.

\section{Proof of Theorem 5}

Define by $\Sigma$ the spectrum of problem

$$
\begin{aligned}
-\Delta_{p} u(x) & =\lambda|u|^{p-2} u \quad \text { in } \Omega, \\
u & =0 \quad \text { on } \gamma, \text { where } \gamma \subset \partial \Omega, \\
\frac{\partial u}{\partial_{p} \nu} & \equiv|\nabla u|^{p-2}(\nabla u, \nu)=0 \quad \text { on } \partial \Omega \backslash \bar{\gamma} .
\end{aligned}
$$

That is, $\Sigma=\{\lambda \in \mathbb{R}$ : the problem (69) has the nontrivial weak solution\}.

Theorem 12. The spectrum $\Sigma$ is nonempty closed set, $\Sigma \subset$ $(0, \infty)$.

Proof. To show that the spectrum is nonempty, one needs to prove the existence of weak solution to problem (69). We can make use of Theorem 7. We take $V=W^{1, p}(\Omega, \gamma)$ and 
$A(u)=-\sum_{i}\left(\partial / \partial x_{i}\right)\left(\left|\partial u / \partial x_{i}\right|^{p-2}\left(\partial u / \partial x_{i}\right)\right)$. For $u, v \in W^{1, p}(\Omega$, $\gamma)$ we define

$$
\begin{aligned}
(A(u), v) & =-\int_{\Omega} \sum_{i} \frac{\partial}{\partial x_{i}}\left(\left|\frac{\partial u}{\partial x_{i}}\right|^{p-2} \frac{\partial u}{\partial x_{i}}\right) v d x \\
& =\int_{\Omega} \sum_{i}\left|\frac{\partial u}{\partial x_{i}}\right|^{p-2} \frac{\partial u}{\partial x_{i}} \frac{\partial v}{\partial x_{i}} d x .
\end{aligned}
$$

One needs to verify the properties of operator $A$. First, the boundedness is fulfilled since

$$
(A(u), u) \leq C\|u\|_{W^{1, p}}^{p} .
$$

To check the semicontinuity, monotonicity of the operator, and the property $(A(u), u) /\|u\| \rightarrow+\infty$ as $\|u\|_{W^{1, p}} \rightarrow+\infty$ one can in the same way as in the proof of Theorem 4 . Let us omit the details.

As soon as we conclude that the conditions of Theorem 7 are fulfilled, we conclude the existence of the solution. Thus, the spectrum is nonempty. Check now the positiveness of $\Sigma$. Let $\lambda \in \Sigma$ and the corresponding eigenfunction $u \in$ $W^{1, p}(\Omega, \gamma)$. It yields that

$$
\begin{aligned}
& \int_{\Omega}|\nabla u|^{p} d x=\lambda \int_{\Omega}|u|^{p} d x, \\
& \text { therefore } \lambda=\frac{\int_{\Omega}|\nabla u|^{p} d x}{\int_{\Omega}|u|^{p} d x}>0 .
\end{aligned}
$$

Let $\lambda_{k} \in \Sigma$ correspond to eigenfunctions $u_{k}$ and $\lambda_{k} \rightarrow \lambda$. Without loss of generality one may assume that $\left\|u_{k}\right\|_{L_{p}}=1$. Since $\left\{\lambda_{k}\right\}$ is bounded, it follows from

$$
\int_{\Omega}\left|\nabla u_{k}\right|^{p} d x=\lambda_{k} \int_{\Omega}\left|u_{k}\right|^{p} d x
$$

that $\left\|u_{k}\right\|_{W^{1, p}(\Omega, \gamma)} \leq C$ which implies the existence of $u \in$ $W^{1, p}(\Omega, \gamma)$ such that up to a subsequence

$$
\begin{aligned}
& u_{k} \rightarrow u \text { weakly in } W^{1, p}(\Omega, \gamma), \\
& u_{k} \longrightarrow u \text { strongly in } L_{p}(\Omega) .
\end{aligned}
$$

Moreover,

$$
\left\|A\left(u_{k}\right)\right\|=\left(A\left(u_{k}\right), u_{k}\right) \leq C\left\|u_{k}\right\|_{L_{p}}^{p-1},
$$

and therefore there exists $\chi$ such that

$$
A\left(u_{k}\right) \rightarrow \chi \text { weakly in } W^{-1, q}(\Omega, \gamma) .
$$

These convergences imply that $\|u\|_{L_{p}}=1$ and

$$
\int_{\Omega} \chi \nabla v d x=\lambda \int_{\Omega}|u|^{p-2} u v d x \quad \text { for } v \in W^{1, p}(\Omega, \gamma) .
$$

Thus it remains to show that

$$
\int_{\Omega} \chi \nabla v d x=\int_{\Omega}|\nabla u|^{p-2} \nabla u \nabla v d x
$$

For every $v \in W^{1, p}(\Omega, \gamma)$ it holds that

$$
\begin{aligned}
0 \leq & \int_{\Omega}\left(\left|\nabla u_{k}\right|^{p-2} \nabla u_{k}-|\nabla v|^{p-2} \nabla v, \nabla u_{k}-\nabla v\right) d x \\
= & \int_{\Omega} \lambda_{k}\left|u_{k}\right|^{p-2} u_{k}\left(u_{k}-v\right) d x \\
& -\int_{\Omega}|\nabla v|^{p-2} \nabla v\left(\nabla u_{k}-\nabla v\right) d x
\end{aligned}
$$

Passing to the limit as $k \rightarrow \infty$, one obtains that

$$
\begin{aligned}
0 \leq & \int_{\Omega} \lambda|u|^{p-2} u(u-v) d x \\
& -\int_{\Omega}|\nabla v|^{p-2} \nabla v(\nabla u-\nabla v) d x \\
= & \int_{\Omega} \chi(\nabla u-\nabla v) d x-\int_{\Omega}|\nabla v|^{p-2} \nabla v(\nabla u-\nabla v) d x .
\end{aligned}
$$

Take now $v=u-t w, w \in W^{1, p}(\Omega, \gamma), t \in \mathbb{R}_{+}$. Then

$$
0 \leq \int_{\Omega}\left(\chi-|\nabla(u-t w)|^{p-2} \nabla(u-t w)\right) \nabla w d x
$$

Passing to the limit as $t \rightarrow+0$, we get

$$
0 \leq \int_{\Omega}\left(\chi-|\nabla u|^{p-2} \nabla u\right) \nabla w d x
$$

From this inequality we deduce the validity of (78).

Let us mention the properties of the first eigenvalue to spectral problem (69). The following theorem directly follows from the results in [34].

Theorem 13. The smallest eigenvalue

$$
\lambda^{1}:=\inf _{u \in W^{1, p}(\Omega, \gamma) \backslash\{0\}} \frac{\int_{\Omega}|\nabla u|^{p} d x}{\int_{\Omega}|u|^{p} d x}
$$

is simple and isolated. Moreover, if $u_{1}, u_{2}$ are two eigenfunctions corresponding to $\lambda^{1}$, then they are proportional: $u_{1}=k u_{2}$.

Now we come back to our spectral problems (3) and (5). The existence of normalized solution and properties of spectrum follows by Theorems 12 and 13. The uniqueness of the normalized solution is a direct consequence of Theorem 13 . Concerning the regularity, the known fact (see [40]) says that if eigenfunctions $u \in W_{0}^{1, p}$, then $u \in L^{\infty}$. The statements of Theorem 5 can be proved similarly to the proof of statement (2) of Theorem 4.

Indeed, since $\lambda_{\varepsilon}^{1}$ is isolated, that is, $\exists \delta>0:\left|\lambda_{\varepsilon}^{1}-\lambda_{\varepsilon}^{2}\right|>\delta$ $\left(\lambda_{\varepsilon}^{2}\right.$ is the second eigenvalue to (3)), there exists a compact containing $\lambda_{\varepsilon}^{1}$. Hence, there exists a subsequence $\left\{\varepsilon_{k}\right\}_{k=1}^{\infty} \rightarrow 0$ such that $\lambda_{\varepsilon_{k}}^{1} \rightarrow \lambda^{*}$. The boundedness of $u_{\varepsilon_{k}}$ in $L_{p}$ implies the existence of limit $u^{*}$ (strong in $L_{p}$ and weak in $W^{1, p}$ ). 
The boundedness of operator $A\left(u_{\varepsilon_{k}}\right)$ in the dual space $W^{-1, q}$ gives the existence of weak limit

$$
\begin{aligned}
\chi & =\lim _{k \rightarrow \infty} A\left(u_{\varepsilon_{k}}\right) \\
& =\lim _{k \rightarrow \infty} \frac{\partial}{\partial x_{j}}\left(\sum_{j=1}^{n}\left|\nabla u_{\varepsilon_{k}}(x)\right|^{p-2} \frac{\partial u_{\varepsilon_{k}}(x)}{\partial x_{j}}\right) .
\end{aligned}
$$

Thus, passing to the limit in the identity

$$
\begin{gathered}
\int_{\Omega} \sum_{j=1}^{n}\left|\nabla u_{\varepsilon_{k}}(x)\right|^{p-2} \frac{\partial u_{\varepsilon_{k}}(x)}{\partial x_{j}} \frac{\partial v(x)}{\partial x_{j}} d x \\
=\lambda_{\varepsilon_{k}}^{1} \int_{\Omega}\left|u_{\varepsilon_{k}}\right|^{p-2} u_{\varepsilon_{k}} v d x,
\end{gathered}
$$

we obtain

$$
\begin{gathered}
\int_{\Omega} \sum_{j=1}^{n}\left|\nabla u^{*}(x)\right|^{p-2} \frac{\partial u^{*}(x)}{\partial x_{j}} \frac{\partial v(x)}{\partial x_{j}} d x \\
=\lambda^{*} \int_{\Omega}\left|u^{*}\right|^{p-2} u^{*} v d x .
\end{gathered}
$$

Having in mind the same arguments as in the proof of Theorem 4 one can show that $\lambda^{*}=\lambda_{0}^{1}, u^{*}=u_{0}$, and $\chi=$ $A\left(u_{0}\right)=-\sum_{i}\left(\partial / \partial x_{i}\right)\left(\left|\partial u_{0} / \partial x_{i}\right|^{p-2}\left(\partial u_{0} / \partial x_{i}\right)\right)$ which means that problem (5) is the limit one for (3). Analogously as in Theorem 4 one can derive that

$$
\left\|u_{\varepsilon_{k}}-u_{0}\right\|_{W^{1, p}} \longrightarrow 0 .
$$

Thus, Theorem 5 is completely proved.

The question on convergence of the full spectrum for similar $p$-Laplace boundary-value problem is studied, for example, in $[33,35]$. We do not cover this studying for our problem since in the applications of the results of the present paper we will use only the fact about converging of the first eigenelements.

\section{The Estimate of the Solution in a Neighborhood of the Eigenvalue}

Let us derive an estimate for the solution to problem (7) in a neighborhood of $\lambda_{0}^{1}$.

Lemma 14. Let $\lambda$ be close to $\lambda_{0}^{1}$ and $\lambda_{\varepsilon}^{1}$ converges to $\lambda_{0}^{1}$. Then the following estimate holds:

$$
\left\|U_{\varepsilon}\right\|_{W^{1, p}}^{p} \leq C \frac{\|F\|_{L_{q}}^{2}}{\left|\lambda_{\varepsilon}^{1}-\lambda\right|} .
$$

Proof. Since the function $U_{\varepsilon} \in W^{1, p}\left(\Omega_{\varepsilon}, \Gamma_{\varepsilon}\right)$, it satisfies Friedrichs inequality $\left\|U_{\varepsilon}\right\|_{L_{p}}^{p} \leq K\left\|\nabla U_{\varepsilon}\right\|_{L_{p}}^{p}$. Moreover, it is clear that the best constant in the inequality is $1 / \lambda_{\varepsilon}^{1}$, where $\lambda_{\varepsilon}^{1}$ is the first eigenvalue to (3). Thus,

$$
\left\|U_{\varepsilon}\right\|_{L_{p}}^{p} \leq \frac{1}{\lambda_{\varepsilon}^{1}}\left\|\nabla U_{\varepsilon}\right\|_{L_{p}}^{p}
$$

By using estimates (43), (8), (89), integral identity with $v=$ $U_{\mathcal{\varepsilon}}$, and equivalent norm in $W^{1, p}$, it yields that

$$
\begin{aligned}
&\left\|\nabla U_{\varepsilon}\right\|_{L_{p}}^{p} \leq \lambda\left\|U_{\varepsilon}\right\|_{L_{p}}^{p}+\|F\|_{L_{p}}\left\|U_{\varepsilon}\right\|_{L_{p}} \\
& \leq \frac{\lambda}{\lambda_{\varepsilon}^{1}}\left\|\nabla U_{\varepsilon}\right\|_{L_{p}}^{p}+\|F\|_{L_{q}}^{2} \\
& \Longrightarrow\left|\lambda_{\varepsilon}^{1}-\lambda\right|\left\|\nabla U_{\varepsilon}\right\|_{L_{p}}^{p} \leq C\|F\|_{L_{q}}^{2}, \\
& \text { where }\left|\lambda_{\varepsilon}^{1}\right|<C=\text { const. }
\end{aligned}
$$

Replacing $\left\|\nabla U_{\varepsilon}\right\|_{L_{p}}^{p}$ with the equivalent norm $\left\|U_{\varepsilon}\right\|_{W^{1, p}}^{p}$, we obtain (88).

\section{Conflict of Interests}

The author declares that there is no conflict of interests regarding the publication of this paper.

\section{Acknowledgments}

The work was partially supported by RFBR (Project 15-5845142) and by the grant of President of Russian Federation supporting young Russian scientists (Project M-4615.2015.1).

\section{References}

[1] B. Amaziane, S. Antontsev, L. Pankratov, and A. Piatnitski, "Homogenization of $p$-laplacian in perforated domain," Annales de l'Institut Henri Poincaré Analyse Non Linéaire, vol. 26, no. 6, pp. 2457-2479, 2009.

[2] N. M. Arató, "Limit boundary value problems in regions with random fine grained boundaries," Applied Mathematics Letters, vol. 8, no. 4, pp. 1-6, 1995.

[3] A. G. Belyaev, On singular perturbations of boundary-value problems [Ph.D. thesis], Moscow State University, Moscow, Russia, 1990 (Russian).

[4] A. G. Belyaev, "Homogenization of a boundary-value problem with a third boundary condition for Poisson equation in a domain perforated along the boundary," Uspekhi Matematicheskikh Nauk, vol. 45, no. 4, p. 123, 1990 (Russian).

[5] G. A. Chechkin, "On boundary-value problems for a secondorder elliptic equation with oscillating boundary conditions," in Nonclassical Partial Differential Equations, V. N. Vragov, Ed., pp. 95-104, Institute of Mathematics, Siberian Division of the Academy of Sciences of the USSR (IM SOAN SSSR), Novosibirsk, Russia, 1988.

[6] G. A. Chechkin, "Asymptotic expansions of the eigenvalues and eigenfunctions of an elliptic operator in a domain with many 'light' concentrated masses near the boundary. The twodimensional case," Izvestiya Rossijskoj Akademii Nauk Seriya Matematicheskaya, vol. 69, no. 4, pp. 161-204, 2005, English Translation: Izvestiya: Mathematics, vol. 69, no. 4, 805-846, 2005.

[7] G. A. Chechkin, T. P. Chechkina, C. D’Apice, and U. De Maio, "Homogenization in domains randomly perforated along the boundary," Discrete and Continuous Dynamical Systems Series $B$, vol. 12, no. 4, pp. 713-730, 2009. 
[8] G. A. Chechkin, Y. O. Koroleva, A. Meidell, and L.-E. Persson, "On the Friedrichs inequality in a domain perforated aperiodically along the boundary. Homogenization procedure. Asymptotics for parabolic problems," Russian Journal of Mathematical Physics, vol. 16, no. 1, pp. 1-16, 2009.

[9] G. A. Chechkin, Y. O. Koroleva, and L.-E. Persson, "On the precise asymptotics of the constant in Friedrich's inequality for functions vanishing on the part of the boundary with microinhomogeneous structure," Journal of Inequalities and Applications, vol. 2007, Article ID 34138, 13 pages, 2007.

[10] G. A. Chechkin, Y. O. Koroleva, L.-E. Persson, and P. Wall, "A new weighted Friedrichs-type inequality for a perforated domain with a sharp constant," Eurasian Mathematical Journal, vol. 2, no. 1, pp. 81-103, 2011.

[11] D. Cioranescu and F. Murat, "Un terme etrange venu d'ailleurs I,II," in Nonlinear Partial Differential Equations and Their Applications: College de France Seminar, vol. 70 of Research Notes in Mathematics 60, no. 2-3, pp. 98-178, 1982.

[12] R. R. Gadyl'shin, "Characteristic frequencies of bodies with thin pipes. Convergence and estimates," Mathematical Notes, vol. 54, no. 6, pp. 1192-1199, 1993.

[13] R. R. Gadyl'shin, "On the asymptotics of the eigenvalues for periodically clamped membranes," St. Petersburg Mathematical Journal, vol. 1, no. 10, pp. 1-14, 1999.

[14] R. R. Gadyl'shin, “The method of matching of asymptotic expansions in a singularly-perturbed boundary-value problem for the Laplace operator," Sovremennaya Matematika $i$ ee Prilozheniya, vol. 125, no. 5, pp. 3-32, 2003, English Translation: Journal of Mathematical Sciences, vol. 125, no. 5, pp. 579-609, 2005.

[15] G. A. Chechkin, R. R. Gadyl'shin, and Y. O. Koroleva, "On the convergence of solutions and eigenelements of a boundary value problem in a domain perforated along the boundary," Differential Equations, vol. 46, no. 5, pp. 667-680, 2010 (Russian), Translation from: Differentsial'nye Uravneniya, vol. 46, no. 5, pp. 665-677, 2010.

[16] G. A. Iosif'yan, O. A. Oleinik, and A. S. Shamaev, "Asymptotic expansions of solutions of the Dirichlet problem for elliptic equations and systems for the theory of elastity in a perforated domain," Doklady Akademii Nauk SSSR, vol. 284, no. 5, pp. 1062-1066, 1985 (Russian).

[17] G. A. Iosif'yan, O. A. Oleinik, and A. S. Shamaev, "On eigenvalues of boundary value problems for a system of the theory of elasticity with rapidly oscillating coefficients in a punctured domain," Matematicheskii Sbornik, vol. 132, no. 4, pp. 517-530, 1987, English Translation: Mathematics of the USSR-Sbornik, vol. 60, no. 2, pp. 505-519, 1988.

[18] Y. O. Koroleva, "On a Friedrichs-type inequality in a threedimensional domain aperiodically perforated along a part of the boundary," Russian Mathematical Surveys, vol. 65, no. 4, pp. 788-790, 2010 (Russian), Translation from: Uspekhi Matematicheskikh Nauk, vol. 65, no. 4, pp. 199-200, 2010.

[19] Y. O. Koroleva, "The Friedrichs inequality in perforated domains. Applications to homogenization theory," in Books of Abstract of International Scientific conference, Ufa, April 30th, 2015, pp. 1-5, 2015.

[20] S. A. Lamonov, "Convergence of solutions of the first boundaryvalue problem for quasilinear parabolic equations in domains with a finegrained boundary," Matematicheskaja Fizika, Nelinejnaja Mekhanika, vol. 36, no. 2, pp. 60-63, 1984 (Russian).

[21] D. V. Larin, "A degenerate quasi-linear Dirichlet problem for domains with a fine-grained boundary. The case of surface distribution of 'grains"' Trudy Instituta Prikladnoi Matematiki i Mekhaniki, vol. 2, pp. 104-115, 1998 (Russian).

[22] M. Lobo, O. A. Oleinik, M. E. Pérez, and T. A. Shaposhnikova, "On homogenization of solutions of boundary value problems in domains, perforated along manifolds," Annali della Scuola Normale Superiore di Pisa. Classe di Scienze. Serie IV, vol. 25, no. 3-4, pp. 611-629, 1997.

[23] V. A. Marchenko and E. Y. Khruslov, Boundary-Value Problems in Domains with Fine-Grained Boundaries, Naukova Dumka, Kiev, Ukraine, 1974, (Russian).

[24] V. A. Marchenko and E. Y. Khruslov, Homogenized Models of Microinhomogeneous Media, Naukova Dumka, Kiev, Ukraine, 2005, English Translation: Homogenization of Partial Differential Equations, vol. 46 of Progress in Mathematical Physics, Birkhäuser, Boston, Mass, USA, 2006.

[25] O. A. Oleinik, A. S. Shamaev, and G. A. Yosifian, "Homogenization of eigenvalues and eigenfunctions of the boundary value problems in perforated domains for elliptic equations with nonuniformly oscillating coefficients," in Current Topics in Partial Differential Equations, pp. 187-216, Kinokuniya, Tokyo, Japan, 1986.

[26] O. A. Oleinik, A. S. Shamaev, and G. A. Yosifian, "Asymptotic expansions of solutions of the Dirichlet problem for elliptic equations in perforated domains," in Nonlinear Partial Differential Equations and Their Applications. Collège de France Seminar, Vol. VIII (Paris, 1984-1985), Pitman Research Notes in Mathematics Series 166, pp. 141-175, Longman Scientific and Technical, Harlow, UK, 1988.

[27] O. A. Oleinik, A. S. Shamaev, and G. A. Yosifian, Mathematical Problems in Elasticity and Homogenization, vol. 26 of Studies in Mathematics and Its Applications, North-Holland, Amsterdam, The Netherlands, 1992.

[28] A. L. Pyatnitskii, A. S. Shamaev, and G. A. Chechkin, Homogenization: Methods and Applications, Tamara Rozhkovskaya, Novosibirsk, Russia, 2007, (Russian).

[29] E. Sánchez-Palencia, "Boundary value problems in domains containing perforated walls," in Nonlinear Partial Differential Equations and Their Applications: College de France Seminar, vol. 70 of Research Notes in Mathematics, no. 3, pp. 309-325, 1982.

[30] I. V. Skrypnik, Methods of Analysis of Nonlinear Elliptic Boundary-Value Problems, Fizmatlit, Moscow, Russia, 1990, English Translation: vol. 139, American Mathematical Society, Providence, RI, USA, 1994.

[31] I. V. Skrypnik, "Asymptotic behaviour of solutions of nonlinear elliptic problems in punctured domains," Matematicheskii Sbornik, vol. 184, no. 10, pp. 67-90, 1993, English Translation: Russian Academy of Sciences. Sbornik Mathematics, vol. 80, no. 1, pp. 191-209, 1993.

[32] I. V. Skrypnik, "New conditions for averaging of nonlinear dirichlet problems in perforated domains," Ukrainskiy Matematicheskiy Zhurnal, vol. 48, no. 5, pp. 675-694, 1996, English Translation: Ukrainian Mathematical Journal, vol. 48, no. 5, pp. 753-774, 1996.

[33] I. V. Skrypnik and Y. V. Namleeva, "Convergence of eigenvalues and eigenfunctions of nonlinear dirichlet problems in domains with fine-grain boundary," Ukrainian Mathematical Journal, vol. 55, no. 6, pp. 993-1011, 2003.

[34] J. F. Bonder, J. P. Pinasco, and A. M. Salort, "Quasilinear eigenvalues," Revista de la Unión Matemática Argentina, vol. 56, no. 1, pp. 1-25, 2015. 
[35] J. F. Bonder, J. P. Pinasco, and A. M. Salort, "Convergence rate for some quasilinear eigenvalues homogenization problems," Journal of Mathematical Analysis and Applications, vol. 423, no. 2, pp. 1427-1447, 2015.

[36] M. Cuesa, "Eigenvalue problems for p-Laplacian with indefinite weights," Electronic Journal of Differential Equations, vol. 2001, no. 33, pp. 1-9, 2001.

[37] P. Lindqvist, "On the equation $\operatorname{div}\left(|\nabla u|^{p-2} \nabla u\right)+\lambda|u|^{p-2} u=0$," Proceedings of the American Mathematical Society, vol. 109, no. 1, pp. 157-164, 1990.

[38] P. Lindqvist, "On the equation $\operatorname{div}\left(|\nabla u|^{p-2} \nabla u\right)+\lambda|u|^{p-2} u=$ 0," Proceedings of the American Mathematical Society, vol. 109, pp. 157-164, 1990, Addendum in: Proceedings of the American Mathematical Society, vol. 116, pp. 583-584, 1992.

[39] J. L. Lions, Some Methods of Solving of Nonlinear BoundaryValue Problems, Mir, Moscow, Russia, 1972 (Russian).

[40] M. Guedda and L. Vèron, "Quasilinear elliptic equations involving critical Sobolev exponents," Nonlinear Analysis: Theory, Methods \& Applications, vol. 13, no. 8, pp. 879-902, 1989. 


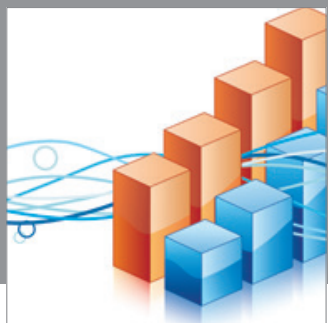

Advances in

Operations Research

mansans

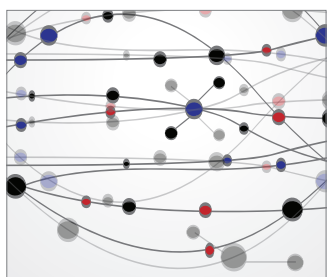

The Scientific World Journal
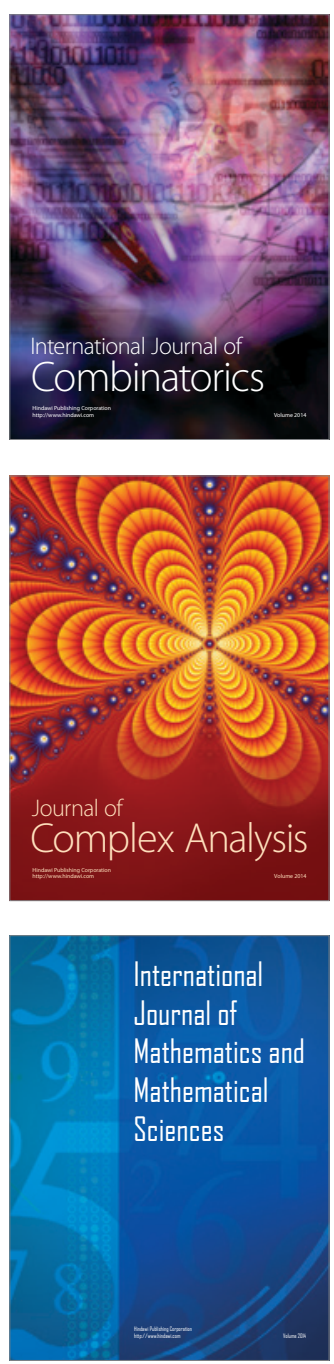
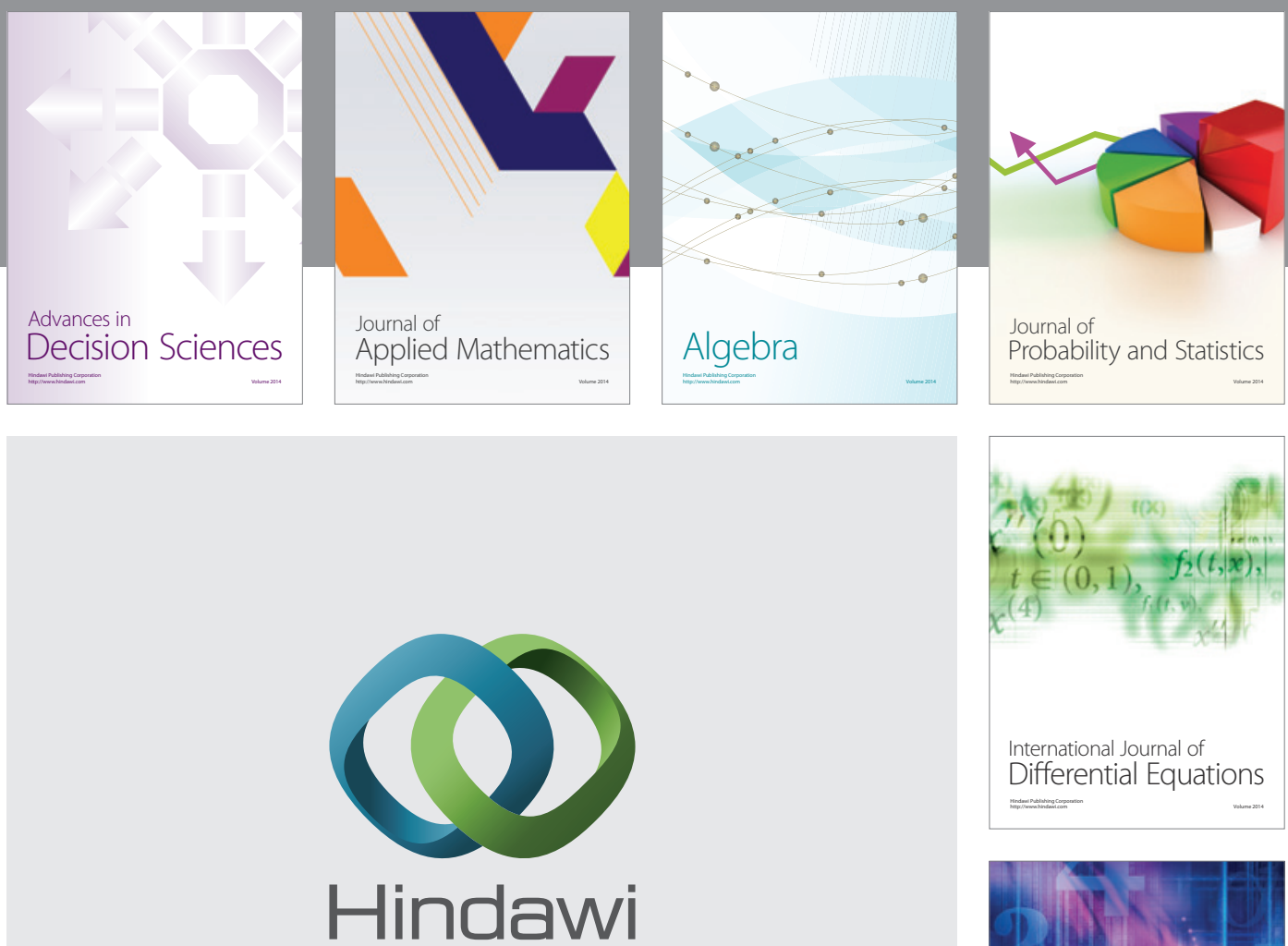

Submit your manuscripts at http://www.hindawi.com
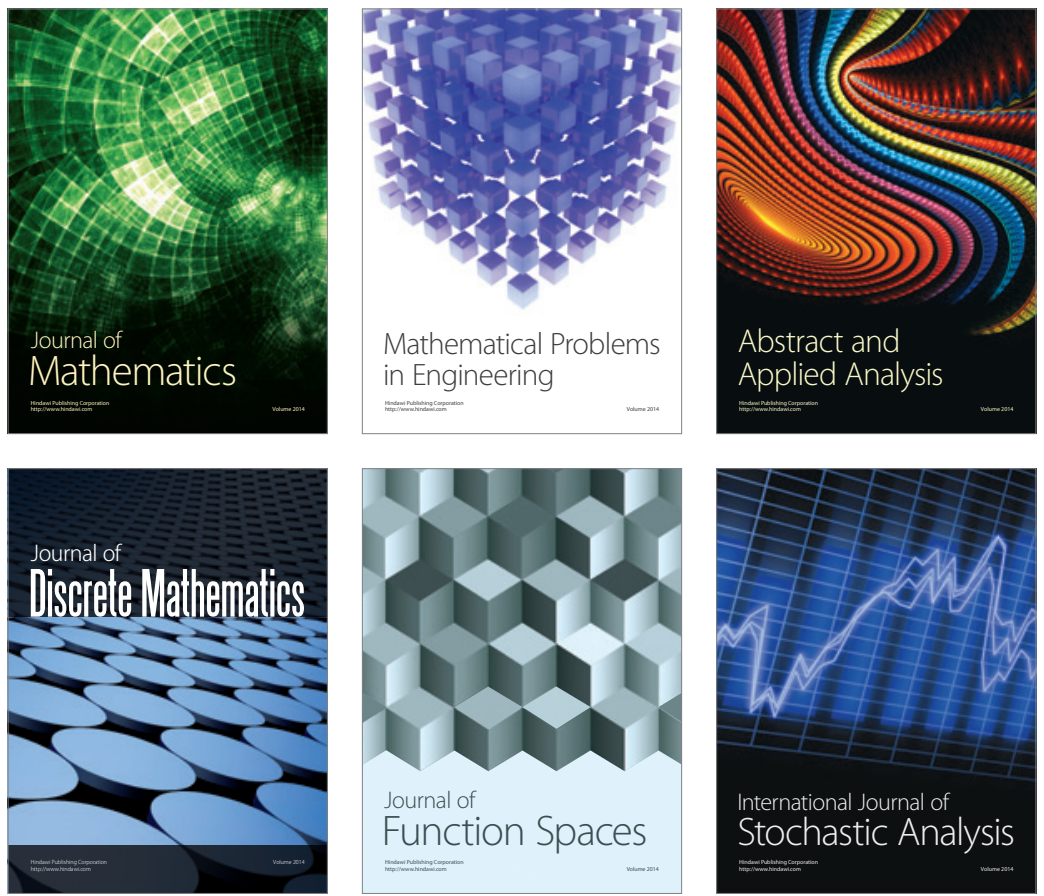

Journal of

Function Spaces

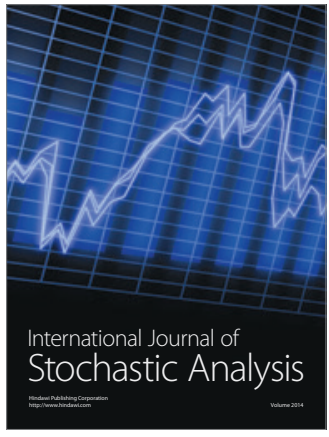

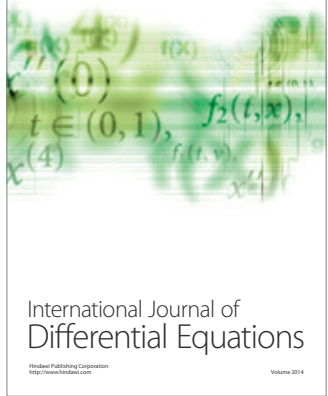
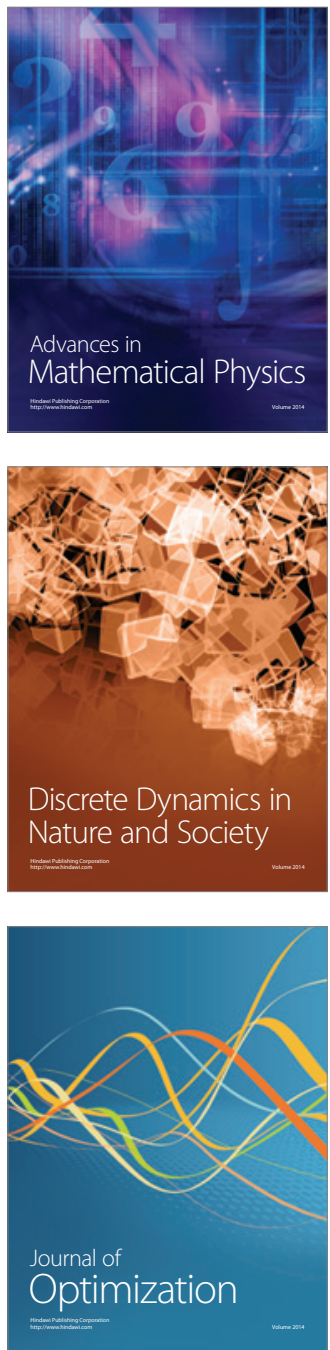